\title{
Shedding light on low-mass subhalo survival with numerical simulations
}

\section{A. Aguirre-Santaella, ${ }^{a, b, *}$ M. A. Sánchez-Conde, ${ }^{a, b}$ R. Angulo, ${ }^{c, d}$ G. Ogiya ${ }^{e, f}$ and J. Stücker ${ }^{c}$}

${ }^{a}$ Instituto de Física Teórica UAM-CSIC, Universidad Autónoma de Madrid, C/ Nicolás Cabrera, 13-15, 28049 Madrid, Spain

${ }^{b}$ Departamento de Física Teórica, M-15, Universidad Autónoma de Madrid, E-28049 Madrid, Spain

${ }^{c}$ Donostia International Physics Center (DIPC),

Manuel Lardizabal Ibilbidea, 4, 20018 Donostia, Gipuzkoa, Spain

${ }^{d}$ IKERBASQUE, Basque Foundation for Science, 48013, Bilbao, Spain

${ }^{e}$ Waterloo Centre for Astrophysics, University of Waterloo, Waterloo, ON N2L 3G1, Canada

${ }^{f}$ Department of Physics and Astronomy, University of Waterloo, 200 University Avenue West, Waterloo, Ontario N2L 3G1, Canada

E-mail: alejandra.aguirre@uam.es, miguel.sanchezconde@uam.es, reangulo@dipc.org, gogiya@uwaterloo.ca, jstuecker@dipc.org

In this work, we carry out a suite of specially-designed numerical simulations to shed further light on dark matter (DM) subhalo survival at mass scales relevant for gamma-ray DM searches, a topic subject to intense debate nowadays. Specifically, we have employed an improved version of DASH, a GPU N-body code, to study the evolution of low-mass subhalos inside a Milky Way-like halo with unprecedented accuracy. We have simulated subhalos with varying mass, concentration, and orbital properties, and considered the effect of the gravitational potential of the Milky-Way galaxy itself. In addition to shedding light on the survival of low-mass galactic subhalos, our results will provide detailed predictions that will aid current and future quests for the nature of DM.

$37^{\text {th }}$ International Cosmic Ray Conference (ICRC 2021)

July 12 th - 23rd, 2021

Online - Berlin, Germany

\footnotetext{
${ }^{*}$ Presenter
} 


\section{Introduction}

There is strong evidence to believe that most of the matter in our Universe is dark [1]. This dark matter (DM) has not been detected in laboratories, yet its gravitational effects have been observed from the innermost regions of galaxies to the large-scale structure of the Universe. Different (yet complementary) approaches are possible for detecting DM: creation in particle accelerators, direct detection of traces of weak interactions between DM and ordinary matter, and indirect detection via DM annihilation/decay products. Indirect detection seeks to detect gamma rays and other Standard Model products that could be produced if DM is a particle that self-annihilates. At present, the gamma-ray sky has already been scrutinized by the Fermi Large Area Telescope (LAT) on board the Fermi satellite [2] and by ground-based Cherenkov telescopes [3]. Yet, no clear and univocal proof of DM has been found [4]. An important open question in DM searches is the role of the so-called DM substructure. In the standard $\Lambda$ CDM cosmological framework, small, dense DM structures (or halos) form first in the early Universe and later merge to form larger structures. A consequence of this hierarchical formation scenario is the existence of abundant substructure (or subhalos) orbiting in halos like that hosting our own Milky-Way (MW) galaxy.

Subhalos hosting dwarf satellite galaxies are known to be excellent targets for gamma-ray DM searches. Less massive subhalos can also be excellent candidates since some of them may be close enough to yield large DM annihilation fluxes [5, 6]. Also, the DM-annihilation flux is proportional to the DM density squared. Thus, the clumpy distribution of subhalos will considerably boost the total DM annihilation in their host halos [7].

Despite its importance, some basic properties of the subhalo population remain uncertain. To follow the complicated dynamics of subhalos, numerical simulations are necessary. Unfortunately, state-of-the-art N-body cosmological simulations do not resolve the whole subhalo hierarchy. Being limited by numerical resolution, they typically simulate subhalos of at least one million solar masses $[8,9]$, i.e. twelve orders of magnitude larger than the minimum halo mass expected in many DM scenarios. Also, finite numerical resolution implies that at least some subhalos will be artificially destroyed in simulations.

Indeed, it is unclear whether small subhalos will survive the strong tidal forces within their hosts since their accretion times to present [10-12]. Some authors claim that almost all subhalo disruption is of numerical origin and a bound remnant should always survive [11,13], while other studies suggest that the abundance of small subhalos is severely reduced due to the effect of tidal forces and of other dynamical agents such as the presence of baryonic material [14, 15]. This on-going debate is of utmost importance for gamma-ray DM searches.

Here, we carry out a suite of specially-designed numerical simulations to shed further light on subhalo survival at all mass scales relevant for DM searches. Specifically, we plan to employ the DASH simulation code [16] to study the evolution of subhalos inside a MW-like halo with unprecedented accuracy. DASH is a fast tree-code optimised for GPU clusters which features both high performance and scalability. DASH simulates the dynamical evolution of subhalos with the N-body method and analytically describes the gravitational potential of the host. In this way, computational resources are focused on a single subhalo, which allows its simulation with extremely high force and mass resolution, which would not be possible in standard cosmological simulations. 


\section{DASH: a code to simulate subhalo evolution}

As stated above, we carry out an ambitious simulation program aimed to shed light on the disruption and survival of subhalos in a gravitational potential similar to that of the MW galaxy. The goal is to characterize the abundance of subhalos of different masses. Since our main scientific driver is to understand the implication of subhalo survival for gamma-ray DM searches, we focus on estimating low-mass subhalo abundances at the solar galactocentric radius. Yet, our simulation programme is general enough to also collect detailed information at broad galactocentric distances, subhalo masses, and cosmic times.

We make use of the DASH code [16] to follow the dynamical evolution of subhalos from their infall times to present, under different configurations of orbits, subhalo masses, and inner structure (characterised by the concentration parameter, which is the ratio between the virial radius of the subhalo and its scale radius, a measure of the subhalo core, where most of the annihilation occurs).

This simulation code has been implemented and optimized for GPU clusters with CUDA GPU toolkit. For computing gravitational forces among particles, DASH uses a hierarchical tree algorithm, that reduces the numerical complexity from $O\left(N^{2}\right)$ to $O(N \log N)$ by grouping particles that are sufficiently far from a given particle and performing a multipole expansion of their gravitational potential. In addition, DASH features the option to include an analytic gravitational potential, with which we will model the effect of the host DM halo mimicking that hosting our Milky-Way galaxy.

These algorithms have been employed in many studies exploring the dynamics of collisionless systems, such as galaxies and DM halos. Due to its intrinsically sequential nature, DASH uses CPUs for constructing the oct-tree structure, but employs GPUs to compute gravitational interaction among particles by traversing the tree structure, which is the most computationally demanding aspect of our problem.

The code has two modes: the first one, treecode, carries out the simulations and outputs snapshots containing the mass, position, velocity, and gravitational potential of each particle. The second one, evolution, analyzes the snapshots and computes the orbital and mass evolution of the subhalo as well as its radial profiles (density, velocity dispersion).

\subsection{Code improvements}

Several important updates have been made to the original DASH code published in [16] for this specific work:

- The host halo DM potential, concentration and virial radius are now evolving with time.

- The galactic disk potential has now been included as well, and it evolves with time according to the recipe in ref. [17]. It consists of three components: the stellar disk, the gas disk, and the bulge. The first two are modeled using Miyamoto-Nagai potentials and the bulge is described with a Hernquist potential.

- A new routine is now in place to select those orbital parameters that lead to a greater probability for a subhalo to cross the solar galactocentric radius. 
- Initial parameters to locate the subhalo can now be selected either via a) initial position and velocity, or b) circularity and orbital energy parameter.

- An option to switch between single and double precision is now available.

\subsection{Parameter choices}

The high numerical accuracy enables us to study with great detail subhalo survival and its impact in gamma-ray DM searches. As the annihilation flux is proportional to the square of the DM density, it is necessary to accurately describe the inner region of each simulated subhalo. Additionally, we simulate subhalos with varying mass, concentration, and orbital properties, thus covering the different properties expected in a realistic cosmological scenario.

We use five parameters to simulate the subhalo:

- The initial subhalo mass, $m_{\text {sub }}$.

- The initial subhalo concentration, $c$.

- The subhalo accretion redshift, $z_{a c c}$.

- The orbit circularity, $\eta=J / J_{\text {circ }}(E)$, where $J$ is the modulus of the angular momentum and $E$ the energy, defined as the total angular momentum in units of the angular momentum for a circular orbit of the same energy $J_{\text {circ }}(E)$ [18].

- The orbital energy parameter, $x_{c}=r_{c i r c}(E) / r$, in units of the radius of a circular orbit of the same energy. Notice that $r$ here is the radius at infall, which is equal to the initial virial radius of the host in our study [18].

These last two are called orbital parameters and have the advantage of depending only on the conserved quantities $E$ and $J$, yet they require adopting a model for the halo potential.

Note that the subhalo is still a halo until the moment of accretion, thus the standard definitions of mass and concentration used for halos are still valid.

\section{Results}

Here, we summarize the current status of our analyses. We have studied two relevant quantities so far: the bound mass fraction, $f_{b}$, which corresponds to the fraction of the initial subhalo mass that remains bound at a given redshift, and the annihilation luminosity, $L_{a n n}$, which is the integral of the subhalo density profile squared.

\subsection{Bound mass fraction}

The bound mass fraction comprises the information about how much mass the subhalo has lost when a certain amount of time has passed since its accretion. In Figure 1, we show $f_{b}$ as a function of the scale factor, $a=1 /(1+z)$, for different subhalo configurations, always adopting a subhalo mass of a million solar masses. We use $2^{18}$ particles and a softening length $\varepsilon=0.304 \mathrm{pc}$. Most significant changes occur at the pericenter, when a larger fraction of material from the subhalo is 
stripped by the host (appearing as abrupt 'steps' in this figure). In particular, in the upper panels, we show the evolution of $f_{b}$ for different concentrations and circularities. We can conclude that less concentrated subhalos at accretion lose mass more quickly. Also, more radial orbits (i.e. those with smaller $\eta$ ) imply a larger mass loss. Note that we are comparing different eccentricities here while fixing $x_{c}$. Therefore, our orbits with higher eccentricities have smaller pericenters and experience a stronger tidal field.

In the lower left panel of Figure 1, we compare the impact of adding baryons to the host potential. As it can be seen, baryonic material can have a huge impact on the mass loss, typically leading to a much smaller $f_{b}$ for the same time after accretion when compared to the non-baryonic case.

Finally, in the lower right panel of Figure 1, we compare runs with different accretion redshifts for both a low and high concentration. These results show that larger accretion redshifts imply a higher number of orbits and larger mass loss. Furthermore, smaller $z_{a c c}$ values induce a larger orbital radius and thus larger orbits.

In Figure 2, we offer a general picture of the $f_{b}$ at $z=0$ for the non-baryonic case, fixing $x_{c}$ to a value of $x_{c}=0.8$ and $z_{a c c}=2$ and varying the concentration and $\eta$ parameters. We obtain, again, that subhalos lose less mass when any of these parameters is larger.

\subsection{Convergence}

We have taken into account two different numerical convergence criteria in order to dilucidate if the subhalo has been physically or numerically disrupted. The first one depends on the softening length, and the second is related to the number of particles [12]. The maximum among the two of these criteria for a given run is the one we finally adopt in each case. We consider that the subhalo has been numerically disrupted and no robust conclusions can be obtained from that simulation if the bound mass fraction drops below that value before $z=0$. We note though that this line lies always below $10^{-2}$, i.e., the subhalo has already lost more than $99 \%$ of its mass by then.

\subsection{Luminosity}

Studying the annihilation luminosity of subhalos is essential to understand their potential for gamma ray searches. The way to compute the luminosity is via the radial density profile $\rho(r)$; more specifically, we define annihilation luminosity in our study as the integration of the profile squared.

The left panel of Figure 3 shows an example of a density profile after the subhalo has been accreted. We observe that the subhalo gets truncated in its outskirts as it orbits around the host and loses mass. The right panel encompasses the luminosity at $z=0$ for a set of concentrations and $\eta$, leaving $x_{c}$ fixed to a value of $x_{c}=0.8$. It can be seen that the concentration is the most relevant parameter, the subhalo losing typically around $40 \%$ of its initial luminosity, yet it can lose up to a $99 \%$ for the lowest $c$ and $\eta$ values considered.

\section{Discussion and conclusions}

Studying subhalo survival is crucial to dilucidate the impact of small subhalos in DM annihilation and indirect searches. This work makes use of DASH, a code specifically designed to perform this task with unprecedent accuracy. Our results show that subhalos with lower concentrations and 

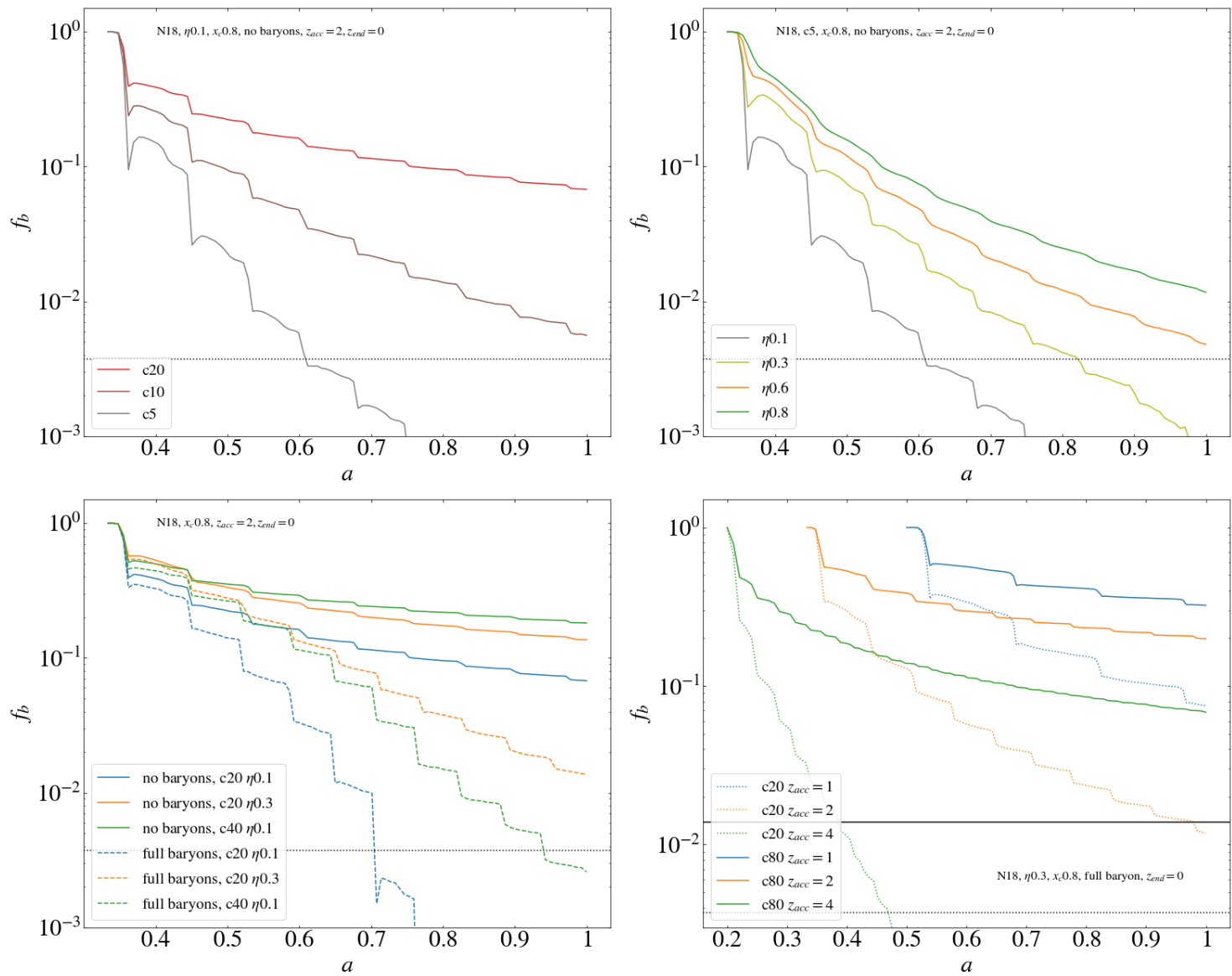

Figure 1: Bound mass fraction as a function of $a=1 /(1+z)$ for different subhalo configurations, always adopting an initial mass of $10^{6} \mathrm{M}_{\text {sun }}$. Upper left panel: Comparison for different initial subhalo concentrations, leaving the orbital energy constant to $x_{c}=0.8$ and only considering a DM host potential. Upper right panel: Comparison for different initial circularities $(\eta)$, leaving the orbital energy constant to $x_{c}=0.8$ and only considering a DM host potential. Lower left panel: Comparison between the case of adding baryons to the host potential according to ref. [17] or using a DM-only potential for two different values of the concentration and orbit circularity. Lower right panel: Comparison among three different accretion redshifts for two different initial subhalo concentrations, for the case of keeping the orbital parameters constant. Black horizontal lines (dotted and solid for the criterion related to number of particles and softening length, respectively) set the convergence value, explained in Section 3.2. All simulations have been run using $2^{18}$ particles and $\varepsilon=0.304$ pc.

subhaloes on orbits with smaller pericenters suffer a larger mass loss. Including baryonic material also induces larger mass loss. Furthermore, the annihilation luminosity depends mainly on the initial subhalo concentration and can get significantly decreased as the subhalo loses mass. We have checked that changing the initial subhalo mass does not have a significant impact on the results, being mass-independent for the DM-only case.

Among potential future applications of our work we can mention, for instance, a more refined calculation of the so-called subhalo boost factor to annihilation signals, more robust constraints on 


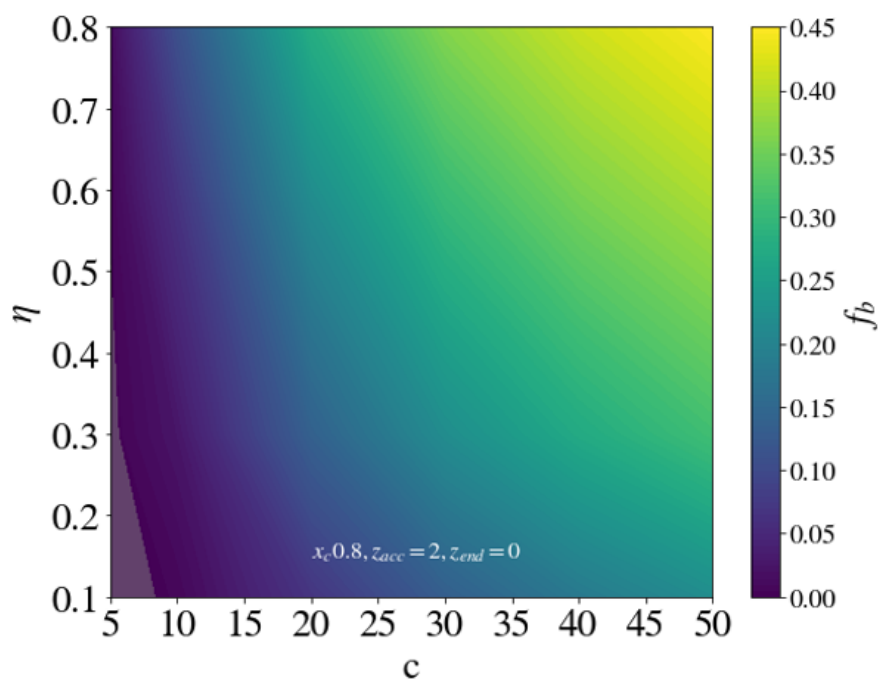

Figure 2: Bound mass fraction at present time for different initial subhalo concentrations and circularities. The shadowed region corresponds to the simulations where convergence could not be achieved. All simulations have been run using $2^{18}$ particles and $\varepsilon=0.304 \mathrm{pc}$.
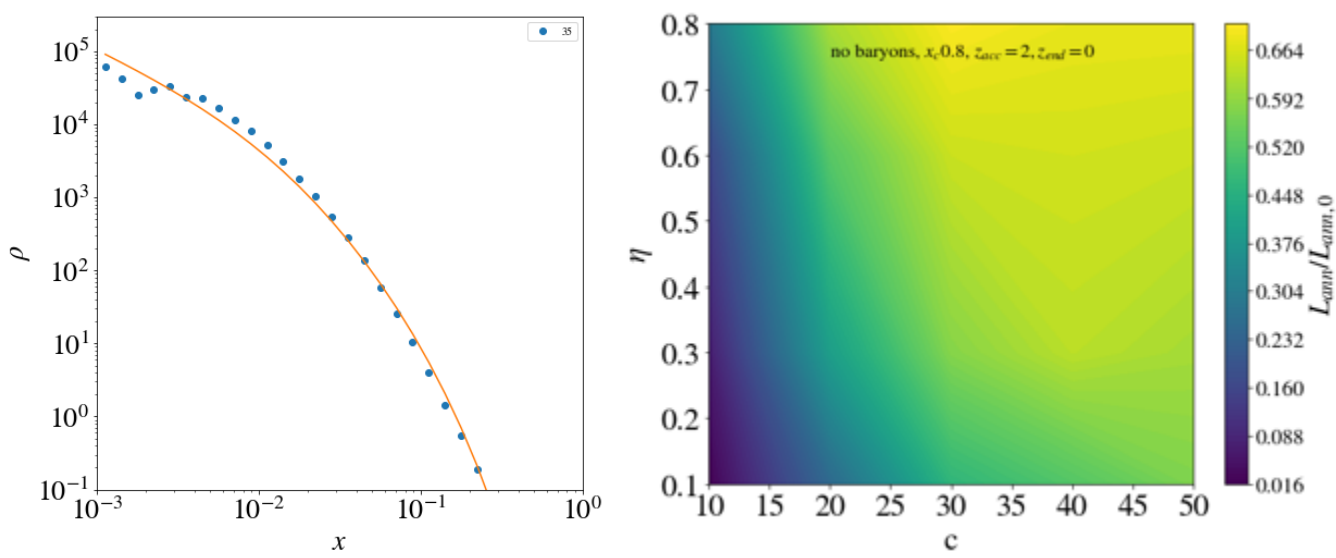

Figure 3: Left panel: Subhalo radial density profile $\rho$ as a function of $x=r / r_{v i r, s}$, i.e. the radius normalized to the initial subhalo virial radius. Right panel: Annihilation luminosity at present time for different initial subhalo concentrations and circularities. All simulations have been run using $2^{18}$ particles and $\varepsilon=0.304 \mathrm{pc}$.

DM, especially for those scientific cases where subhalos play a central role, and the optimization of DM search observation strategies for spatially extended sources.

This work is still ongoing. In the near future, we will expand our parameter space, adding e.g. new values of $x_{c}$. We will also have a closer look at the evolution of the concentration parameter with time and its impact for gamma ray searches.

\section{References}

[1] The Planck Collaboration, Planck 2015 results. XIII. Cosmological parameters, Astron. Astrophys. 594 (2016) 
A13 [1502.01589v3].

[2] N. Gehrels and P. Michelson, GLAST: the next-generation high energy gamma-ray astronomy mission, Astropart. Phys. 11 (1999) 277.

[3] MAGIC collaboration, Status of the 17-m MAGIC telescope, New Astron. Rev. 48 (2004) 339.

[4] J. Conrad, Statistical issues in astrophysical searches for particle dark matter, Astropart. Phys. 62 (2015) 165 [1407.6617].

[5] J. Coronado-Blázquez, M.A. Sánchez-Conde, A. Domínguez, A. Aguirre-Santaella, M.D. Mauro, N. Mirabal et al., Unidentified gamma-ray sources as targets for indirect dark matter detection with the Fermi-Large Area Telescope, JCAP 2019 (2019) 020.

[6] J. Coronado-Blázquez, M.A. Sánchez-Conde, M.D. Mauro, A. Aguirre-Santaella, I. Ciucă, A. Domínguez et al., Spectral and spatial analysis of the dark matter subhalo candidates among Fermi Large Area Telescope unidentified sources, JCAP 2019 (2019) 045.

[7] A. Moliné, M.A. Sánchez-Conde, S. Palomares-Ruiz and F. Prada, Characterization of subhalo structural properties and implications for dark matter annihilation signals, Monthly Notices of the Royal Astronomical Society (2017) stx026.

[8] J. Diemand, M. Kuhlen, P. Madau, M. Zemp, B. Moore, D. Potter et al., Clumps and streams in the local dark matter distribution, Nature (2008) [0805.1244v2].

[9] V. Springel, J. Wang, M. Vogelsberger, A. Ludlow, A. Jenkins, A. Helmi et al., The Aquarius Project: the subhaloes of galactic haloes, Monthly Notices of the Royal Astronomical Society 391 (2008) 1685 [0809.0898].

[10] E. Hayashi, J.F. Navarro, J.E. Taylor, J. Stadel and T. Quinn, The Structural Evolution of Substructure, APJ 584 (2003) 541 [astro-ph/0203004].

[11] F.C. van den Bosch, G. Ogiya, O. Hahn and A. Burkert, Disruption of dark matter substructure: fact or fiction?, MNRAS 474 (2018) 3043 [1711.05276].

[12] F.C. van den Bosch and G. Ogiya, Dark matter substructure in numerical simulations: a tale of discreteness noise, runaway instabilities, and artificial disruption, MNRAS 475 (2018) 4066 [1801. 05427].

[13] R. Errani and J. Peñarrubia, Can tides disrupt cold dark matter subhaloes?, MNRAS 491 (2020) 4591 [1906.01642].

[14] S. Garrison-Kimmel, A. Wetzel, J.S. Bullock, P.F. Hopkins, M. Boylan-Kolchin, C.-A. Faucher-Giguère et al., Not so lumpy after all: modelling the depletion of dark matter subhaloes by Milky Way-like galaxies, MNRAS 471 (2017) 1709 [1701.03792].

[15] R.J.J. Grand and S.D.M. White, Baryonic effects on the detectability of annihilation radiation from dark matter subhaloes around the Milky Way, MNRAS 501 (2021) 3558 [2012.07846].

[16] G. Ogiya, F.C. van den Bosch, O. Hahn, S.B. Green, T.B. Miller and A. Burkert, DASH: a library of dynamical subhalo evolution, MNRAS 485 (2019) 189 [1901.08601].

[17] T. Kelley, J.S. Bullock, S. Garrison-Kimmel, M. Boylan-Kolchin, M.S. Pawlowski and A.S. Graus, Phat ELVIS: The inevitable effect of the Milky Way's disc on its dark matter subhaloes, MNRAS 487 (2019) 4409 [1811.12413].

[18] L. Jiang, S. Cole, T. Sawala and C.S. Frenk, Orbital parameters of infalling satellite haloes in the hierarchical

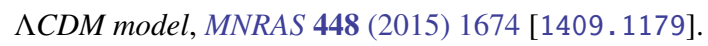

